\title{
Stereocomplex formation in ABA triblock copolymers of poly(lactide) (A) and poly(ethylene glycol) (B)
}

\author{
Willem M. Stevels, Marc J. K. Ankoné, Pieter J. Dijkstra, Jan Feijen* \\ Department of Chemical Technology, University of Twente, P. O. Box 217, 7500 AE \\ Enschede, The Netherlands
}

(Received: February 16, 1995; revised manuscript of March 22, 1995)

\section{SUMMARY:}

Two series of triblock copolymers of poly(ethylene glycol) (PEG, number-average molecular weight $\bar{M}_{\mathrm{n}}=6000$ ) and poly(L-lactide) (PLLA) or poly(D-lactide) (PDLA) were prepared by ring-opening polymerization of lactide initiated by PEG end groups using stannous octoate as a catalyst, either in refluxing toluene or in the melt at $175^{\circ} \mathrm{C}$. The weight percentage of PLA in the polymers varied between 15 and $75 \mathrm{wt} .-\%$. Blends of polymers containing blocks of opposite chirality were prepared by co-precipitation from homogeneous solutions. The melting temperatures of the crystalline PEG and PLA phases strongly depended on the composition of the polymers. The melting temperature of the PLA phase in the blends was approximately $40^{\circ} \mathrm{C}$ higher than that of the single block copolymers. Stereocomplex formation between blocks of enantiomeric poly(lactides) in PEG/PLA block copolymers was established for the first time. Water uptake of polymeric films prepared by solution casting was solely determined by the PEG content of the film.

\section{Introduction}

Block copolymers of poly(lactide) and poly(ethylene glycol) have attracted much attention because they offer a unique combination of biodegradability and hydrophilicity. Poly(lactide)s and random or block copolyesters based on poly(lactide)s have found applications as drug delivery systems and medical sutures ${ }^{1,2)}$. Toxicity of these polymers is intrinsically low because metabolic pathways exist for the main degradation product of these polymers, lactic acid. Poly(ethylene glycol) is a water soluble polymer, which is accepted in most countries as a food or drug additive.

Recently it was shown that biodegradable hydrogels based on photopolymerized poly[(ethylene glycol)-co-lactide] diacrylate macromers have great potential as wound covering material ${ }^{3)}$. Block copolymers based on PEG/PLA could provide a biodegradable alternative to $\mathrm{PEG} /$ poly(ethylene terephthalate) copolymers as a material for the use in surgery ${ }^{4)}$.

Co-crystallization of enantiomeric poly(lactide)s in a racemic lattice, also called stereocomplex formation, leads to materials with higher melting points and lower critical gelation concentrations in solution compared to the optically pure polymers ${ }^{5,6,7)}$. These phenomena have been described extensively ${ }^{8}$. Stereocomplex formation in block copolymers of poly(L-lactide)-block-poly-(D-lactide) was shown for the first time by our group and later by others ${ }^{9,10)}$.

The concepts of PEG/PLLA block copolymers and stereocomplex formation in PLA have now been combined by the preparation of binary blends of PEG/PLLA and PEG/PDLA block copolymers. A series of triblock copolymers was prepared by ring- 
opening polymerization of lactide initiated by PEG end groups in refluxing toluene using stannous octoate as a catalyst. A similar series of polymers was also prepared in the melt at $175^{\circ} \mathrm{C}$. The polymers were characterized using NMR $\left({ }^{1} \mathrm{H},{ }^{13} \mathrm{C}\right)$ spectroscopy and gel-permeation chromatography. Thermal properties were studied by differential scanning calorimetry (DSC). The relative water uptake was determined gravimetrically by immersing films of $300 \mu \mathrm{m}$ thickness in water.

\section{Experimental part}

L-(-)-lactide and D- $(+)$-lactide (Purac Biochem b.v., the Netherlands) were used as received. Poly(ethylene glycol) 6000 (PEG6000, $\bar{M}_{\mathrm{w}} / \bar{M}_{\mathrm{n}}=1.05$ ) (Merck-Schuchardt, Darmstadt Germany) was dried under reduced pressure before use. Stannous ${ }^{\mathrm{II}}$ octoate (stanous ${ }^{\text {Il }}$ 2-ethylhexanoate) (Sigma Chem. Co., St. Louis, USA) was used as received. Toluene was distilled from sodium benzophenoneketyl prior to use. All glassware was dried before use and reactions were carried out in an argon atmosphere. A $0.05 \mathrm{M}$ stock solution of $\mathrm{Sn}(\mathrm{Oct})_{2}$ in toluene was prepared in a Braun $150 \mathrm{GI}$ drybox.

\section{Solution polymerizations}

In a typical experiment a total amount of $10 \mathrm{~g}$ of starting material (PEG6000 and I- or D-lactide) was dissolved in $50 \mathrm{~mL}$ toluene at $70^{\circ} \mathrm{C}$ and an appropriate amount of $\mathrm{Sn}(\mathrm{Oct})_{2}$ solution ( $1 \mathrm{wt} .-\%$ of lactide amount) was added. After refluxing for $24 \mathrm{~h}$, the reaction mixture was cooled, the solvent removed under reduced pressure and the polymer was dried overnight at $40^{\circ} \mathrm{C}$ in vacuo. Before further work-up a sample was taken for NMR and gelpermeation chromatography (GPC) analysis. The polymer was dissolved in $40 \mathrm{~mL}$ of chloroform and precipitated in a five fold excess of cold diethyl ether and dried again.

\section{Melt polymerizations}

In a typical experiment a total amount of $10 \mathrm{~g}$ of starting material (PEG6000 and L- or D-lactide) was mixed with an appropriate amount of $\mathrm{Sn}(\mathrm{Oct})_{2}$ solution $(10 \mathrm{~mol}-\%$ relative to PEG-OH end groups). The mixture was heated to $175^{\circ} \mathrm{C}$ and after $4 \mathrm{~h}$ the reaction mixture was cooled and dissolved in $40 \mathrm{~mL}$ of chloroform. A sample was taken for NMR and GPC analysis. The polymer was precipitated in a five fold excess of cold, diethyl ether and dried overnight at $40^{\circ} \mathrm{C}$ in vacuo.

\section{Blend-and film preparation}

A chloroform solution containing equal amounts of PLLA/PEG and PDLA/PEG polymers $(10 \mathrm{wt} .-\%)$ was stirred for $72 \mathrm{~h}$. The mixture was precipitated in a ten fold excess of well stirred hexane and the polymer thus obtained dried overnight in vacuo. Films were prepared by solution casting of $10 \mathrm{wt} .-\%$ chloroform solutions of the polymer onto silanized dishes. The relative water uptake, defined as the difference between dry and wet weight divided by the dry weight, was determined gravimetrically by immersing films of $300 \mu \mathrm{m}$ thickness in water.

\section{Characterization}

The number-average degree of polymerization of the poly(lactide) blocks was determined from ${ }^{1} \mathrm{H}$ NMR spectra by end group analysis and by comparison to the PEG backbone 
signal. ${ }^{1} \mathrm{H}$ NMR spectra were recorded on a Bruker AC 250 operating at $250 \mathrm{MHz}\left({ }^{1} \mathrm{H}\right)$ or $62.5 \mathrm{MHz}\left({ }^{13} \mathrm{C}\right)$ in chloroform- $d_{1}$. Crude polymers and starting PEG were reacted with trifluoroacetic anhydride to be able to distinguish between reacted and unreacted PEG-OH end groups. To quantitate the number of $-\mathrm{OH}$ groups in the starting PEG a spectrum in DMSO- $d_{6}$ was recorded. Gel-permeation chromatography (GPC) was used to determine molecular weights and molecular weight distributions $\left(\bar{M}_{\mathrm{w}} / \bar{M}_{\mathrm{n}}\right)$. GPC measurements were carried out with tetrahydrofuran (THF) as the eluent $(2.0 \mathrm{~mL} / \mathrm{min})$ using a Waters 510 pump, a HP 1050 autosampler, four Waters $\mu$ Styragel columns $\left(10^{5}, 10^{4}, 10^{3}, 5 \times 10^{2}\right)$ in series, a Waters 410 differential refractometer, and a Viscotek Viscometer Detector H502. The columns were calibrated with polystyrene standards using the universal calibration technique.

Thermal analysis of polymers was carried out with a Perkin-Elmer DSC7 differential scanning calorimeter calibrated with pure indium. The polymers were heated from $-20^{\circ} \mathrm{C}$ to $200^{\circ} \mathrm{C}$ (homopolymers) or $250^{\circ} \mathrm{C}$ (blends) at a heating rate of $20^{\circ} \mathrm{C} / \mathrm{min}$, annealed for $1 \mathrm{~min}$ and cooled to $-20^{\circ} \mathrm{C}$ at a cooling rate of $5^{\circ} \mathrm{C} / \mathrm{min}$ and a second heating curve was recorded.

\section{Results and discussion}

\section{Synthesis and characterization}

Various ways of preparing ABA triblock copolymers of PEG (B) and PLLA (A) have been established. In one study, polymers were prepared by polycondensating lactic acid in the presence of - $\mathrm{OH}$ terminated PEG, but no information on the molecular weight or molecular weight distribution was presented ${ }^{11)}$. Rather well defined polymers are prepared by reaction of $\mathrm{Al}\left(\mathrm{O}^{\mathrm{i} P r}\right)_{3}$ or $\mathrm{Na}$ with $-\mathrm{OH}$ terminated $\mathrm{PEG}$, followed by polymerization of $L$-lactide ${ }^{12,13)}$. In the latter case some racemization of L-lactide was observed. The polymers can even be prepared in the absence of any catalyst, but this requires long reaction times and extremely pure starting materials ${ }^{14)}$. In this case the presence of diblock copolymers was not investigated.

However, the simplest way of preparing well defined triblock copolymers of PEG and PLLA is by using $\mathrm{Sn}(\mathrm{Oct})_{2}$ as a catalyst ${ }^{15,16)}$. $\mathrm{Sn}(\mathrm{Oct})_{2}$ is well known as a catalyst in homopolymerization of lactide and is widely accepted as a food additive or contaminant in PLA for pharmaceutical applications. Therefore, we used this method for the preparation of the ABA block copolymers. If polymers were prepared at $130^{\circ} \mathrm{C}$ in bulk, yields were low, presumably due to fractionation during the purification steps. The GPC trace of such a reaction mixture shows a bimodal distribution, with one of the peaks at an elution volume just above that of the starting PEG. After derivatization with trifluoroacetic anhydride, no peak assignable to a trifluoroacetic ester derivative of a PEG-OH end group can be discerned in the ${ }^{1} \mathrm{H}$ NMR spectrum. It seems that despite the bimodal molecular weight distribution all PEG-OH end groups have initiated lactide polymerization. Probably, phase separation occurs during the synthesis in a PLA/lactide rich and a PEG rich phase, enhancing further chain growth of the longer PLA chains. Previously it was shown that the use of $\mathrm{Sn}(\mathrm{Oct})_{2}$ in xylene allowed the synthesis of poly(D,L-lactide) at temperatures below the melting temperature of the monomer $\mathrm{D}, \mathrm{L}$-lactide ${ }^{17)}$. Therefore, two simple laboratory procedures 
which suppress phase separation were investigated. Reactions were carried out in refluxing toluene and in bulk at $175^{\circ} \mathrm{C}$ (Eq. (1)) and results are shown in Tab. 1.

The NMR analysis of the ABA triblock copolymers of PLLA and PEG was described by other authors and will not be discussed in detail here ${ }^{12,15)}$. The general features of the NMR spectra do not differ from literature data. No unreacted PEG$\mathrm{OH}$ end groups remained after reaction, as shown by ${ }^{1} \mathrm{H}$ NMR spectroscopy after preparation of the trifluoroacetic ester derivative of the polymers. Thus, all PEG-OH end groups have initiated polymerization and no diblock copolymer is present in the reaction mixture. The signal for the carbonyl oxygen atom in the PLA blocks is a sharp singlet, indicating absence of racemization.

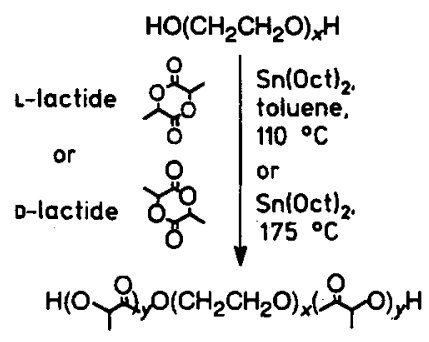

Tab. 1. Results of the polymerization reactions carried out in bulk $\left(175^{\circ} \mathrm{C}\right)$ or in refluxing toluene

\begin{tabular}{|c|c|c|c|c|c|c|c|}
\hline Code $^{a)}$ & $\begin{array}{l}\text { PEG con- } \\
\text { tent in } \\
\text { feed in } \\
\text { wt. }-\%\end{array}$ & $\begin{array}{l}\text { Conv. } \\
\text { in } \%\end{array}$ & $\begin{array}{l}\text { Isolated } \\
\text { yield } \\
\text { in } \%\end{array}$ & $\begin{array}{l}\text { PEG } \\
\text { con- }^{-} \\
\text {tent }^{c)} \\
\text { in wt. } \%\end{array}$ & $\frac{10^{-3} \cdot \bar{M}_{\mathrm{n}, \mathrm{NMR}}}{\mathrm{g} \cdot \mathrm{mol}^{-1}}$ & $\frac{10^{-3} \cdot \bar{M}_{\mathrm{n}, \mathrm{GPC}}}{\mathrm{g} \cdot \mathrm{mol}^{-1}}$ & ${ }_{\underline{C}}^{d)} \bar{M}_{\mathrm{w}} / \bar{M}_{\mathrm{n}}$ \\
\hline LAB1 & 75 & 99 & 82 & 86 & 6.9 & 8.2 & 1.08 \\
\hline LAB2 & 50 & 89 & 84 & 56 & 10.1 & 9.5 & 1.21 \\
\hline LAB3 & 25 & 98 & 82 & 27 & 23.5 & 16.1 & 1.55 \\
\hline DAB1 & 75 & 86 & 83 & 85 & 6.7 & 8.6 & 1.09 \\
\hline DAB2 & 50 & 96 & 82 & 47 & 10.3 & 9.7 & 1.22 \\
\hline DAB3 & 25 & 96 & 82 & 28 & 17.7 & 13.2 & 1.50 \\
\hline LAS1 & 75 & 87 & 92 & 82 & 6.9 & 8.8 & 1.09 \\
\hline LAS2 & 50 & 99 & 91 & 55 & 10.8 & 12.2 & 1.20 \\
\hline LAS3 & 25 & $>99$ & 85 & 28 & 20.5 & 23.1 & 1.41 \\
\hline DAS1 & 75 & 89 & 90 & 83 & 6.8 & 8.8 & 1.08 \\
\hline DAS2 & 50 & 99 & 90 & 55 & 10.8 & 11.6 & 1.17 \\
\hline DAS3 & 25 & $>99$ & 89 & 28 & 20.4 & 18.4 & 1.41 \\
\hline
\end{tabular}

a) LA or DA in a code name refers to $\mathrm{L}$ - or D-lactide monomer, $\mathrm{B}$ or $\mathrm{S}$ refers to polymers prepared in bulk or in solution.

b) Conversion of lactide as determined by ${ }^{1} \mathrm{H}$ NMR spectroscopy.

c) PEG content in the polymer after purification as determined by ${ }^{1} \mathrm{H}$ NMR spectroscopy.

d) Calibrated with polystyrene standards using the universal calibration technique. 
GPC traces for unpurified and purified polymers are similar and show symmetric, unimolecular weight distributions. The polydispersities increase with increasing molecular weight and range between 1.09 and 1.55. No low molecular weight fractions are detected, confirming efficient initiation of the lactide polymerization by the $\mathrm{PEG}-\mathrm{OH}$ end groups. Number average molecular weights determined by GPC and ${ }^{1} \mathrm{H}$ NMR spectroscopy are in good agreement.

Polymers prepared by bulk polymerization at $175^{\circ} \mathrm{C}$ appeared pale yellow in color as reported previously ${ }^{16)}$, while polymers prepared in solution were white. Conversion of lactide was generally high, as were yields, especially for the polymers prepared in solution. The PEG content in the purified polymers did not differ largely from the expected content from the monomer/PEG feed ratio. The agreement was best for the polymers containing longer poly(lactide) blocks. For polymers containing short poly(lactide) blocks the agreement was better for polymers prepared in solution than for those prepared in bulk. In all, well defined triblock copolymers were prepared by both methods. Solution polymerization has the advantage of yielding polymers, at a low polymerization temperature with a composition close to the composition expected from the feed contents, without discoloration.

\section{Thermal properties}

Melting points and melting enthalpies of polymers and blends determined by differential scanning calorimetry are presented in Tab. 2. Second heating curves of polymers with a crystalline poly(lactide) phase are shown in Fig. 1. Glass transition temperatures are not detected because the $T_{\mathrm{g}}$ of the PLA phase coincides with the melting peak of the PEG phase and the $T_{\mathrm{g}}$ of the PEG phase is below the temperature range studied. Attempts to determine glass transition temperatures by dynamic mechanical analysis were hampered by the brittleness of most of the test specimens

Tab. 2. Thermal properties ${ }^{\text {a) }}$ (melting temperatures and melting enthalpies) as determined by DSC of the polymers LAS1 -3 , DAS1 -3 , and the blends LDAS1 -3

\begin{tabular}{|c|c|c|c|c|c|}
\hline & $\begin{array}{l}\text { PEG content } \\
\text { in wt.- } \%\end{array}$ & $\frac{T_{\mathrm{m}, \mathrm{PEG}}}{{ }^{\circ} \mathrm{C}}$ & $\frac{\Delta H_{\mathrm{m}, \mathrm{PEG}}}{\mathrm{J} / \mathrm{g}}$ & $\frac{T_{\mathrm{m}, \text { PLA }}}{{ }^{\circ} \mathrm{C}}$ & $\frac{\Delta H_{\mathrm{PLA}}}{\mathrm{J} / \mathrm{g}}$ \\
\hline PEG6000 & 100 & 64 & 217 & & \\
\hline LAS1 & 82 & 54 & 150 & & \\
\hline LAS2 & 55 & 44 & 104 & 146 & 34 \\
\hline LAS3 & 28 & 42 & 27 & 164 & 47 \\
\hline DAS1 & 83 & 57 & 158 & & \\
\hline DAS2 & 55 & 44 & 107 & 142 & 28 \\
\hline DAS3 & 28 & 40 & 16 & 159 & 43 \\
\hline LDAS1 & 83 & 53 & 136 & & \\
\hline LDAS2 & 55 & 41 & 95 & 182 & 17 \\
\hline LDAS3 & 28 & 44 & 46 & 204 & 63 \\
\hline
\end{tabular}

a) Second heating curves, heating rate $20^{\circ} \mathrm{C} / \mathrm{min}$. 


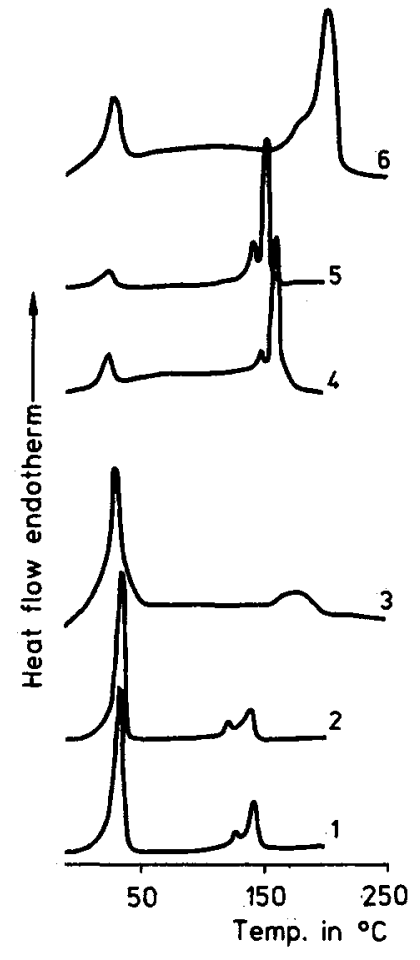

Fig. 1. Second heating curves of the polymers LAS2 (1), DAS2 (2), LDAS2 (3), LAS3 (4), DAS3 (5) and LDAS3 (6) (precipitated material)

prepared by compression moulding. As can be expected for a block copolymer, two melting peaks, each for one polymer phase, are detected. The data for each pair of $\mathrm{L}-$ and D-polymers are very similar, and small differences may reflect slight differences in composition or are within the accuracy of the measurement. Crystallization of the PEG phase occurs in all polymers studied after slow cooling of the block copolymers from the melt, and melting temperature and melting enthalpy decrease with decreasing PEG content. The $T_{\mathrm{m}}$ and $\Delta H_{\mathrm{m}}$ of the polymers having the highest PEG contents (LAS1, DAS1) are still considerably lower than those of the PEG homopolymer, indicating that the presence of a second phase influences the crystallization behaviour to a large extent. Crystallization of the PLA phase does not occur in polymers containing less than 20 wt.- \% PLA, which in this case corresponds to about 14 lactyl units. In poly( $\varepsilon$-caprolactone)-block-poly(L-lactide) polymers of comparable molecular weight the minimum poly(L-lactide) block length for crystallization was found to be around 40 lactyl units ${ }^{18)}$. Polymers containing 60 lactyl units (LAS2 and DAS2) showed crystallinity of the PLA phase. The polymers which contain a crystalline PLA phase, show increasing $T_{\mathrm{m}}$ and $\Delta H_{\mathrm{m}}$ with increasing PLA content.

The DSC traces of the blends differ largely from those of the separate block copolymers. The melting point of the PLA phase is increased by approximately $40^{\circ} \mathrm{C}$, independent of the composition of the polymers. The sharp increase in melting 
temperature has been shown to be related to the crystallization of the PLA blocks in a racemic lattice, or stereocomplex formation ${ }^{5}$. Interestingly, the stereocomplex formation has no pronounced effect on the melting enthalpy in line with observations on blends of PLLA and PDLA ${ }^{6}$. The higher melting point of the crystals in the PLA phase can be attributed to a lower entropy of fusion. Also, the phase behaviour of the PEG phase seems to be unaffected by the stereocomplex formation.

The thermal properties of films prepared by solution casting were determined as well. The results are shown in Tab. 3. The curves of precipitated and solution casted material were very similar when first heating curves are considered. In polymers containing only 28 wt.- $\%$ PEG, no melting endotherm of a PEG phase was detected in the first heating curves of precipitated material or films. In these polymers crystallinity of the PEG phase is completely inhibited by fast crystallization of a continuous PLA phase. Again, high melting racemic crystallites were observed. In all materials studied the melting point of these crystallites was lowered when recording a second heating curve, in contrast to the PLA phase in the unmixed polymers. No satisfactory explanation for this phenomenon can be given presently.

Tab. 3. Thermal properties of films of $300 \mu \mathrm{m}$ thickness compared with precipitated material

\begin{tabular}{|c|c|c|c|c|c|c|c|}
\hline & \multirow{2}{*}{$\begin{array}{l}\text { PEG con- } \\
\text { tent in } \\
\text { wt.- } \%\end{array}$} & \multicolumn{3}{|c|}{ Precipitated materials } & \multirow[b]{2}{*}{$\frac{\Delta H_{\mathrm{PLA}}}{\mathrm{J} / \mathrm{g}}$} & \multicolumn{2}{|c|}{ Films } \\
\hline & & $\begin{array}{c}\frac{T_{\mathrm{m}, \mathrm{PLA}}}{{ }^{\circ} \mathrm{C}} \\
\text { second }^{\mathrm{a})}\end{array}$ & $\begin{array}{l}\frac{T_{\mathrm{m}, \mathrm{PLA}}}{{ }^{\circ} \mathrm{C}} \\
\text { first }^{\mathrm{a})}\end{array}$ & $\begin{array}{c}\Delta H_{\text {PLA }} \\
\mathrm{J} / \mathrm{g} \\
\text { second }\end{array}$ & & $\begin{array}{l}\frac{T_{\mathrm{m}, \text { PLA }}}{{ }^{\circ} \mathrm{C}} \\
\text { first }\end{array}$ & $\frac{\Delta H_{\mathrm{PLA}}}{\mathrm{J} / \mathrm{g}}$ \\
\hline LAS2 & 55 & 146 & 150 & 34 & 36 & 147 & 36 \\
\hline DAS2 & 55 & 142 & 145 & 28 & 38 & 143 & 24 \\
\hline LDAS2 & 55 & 182 & 199 & 17 & 47 & 205 & 61 \\
\hline LAS3 & 28 & 164 & 164 & 47 & 44 & 164 & 50 \\
\hline DAS3 & 28 & 159 & 160 & 43 & 44 & 160 & 50 \\
\hline LDAS3 & 28 & 204 & 219 & 63 & 85 & 220 & 35 \\
\hline
\end{tabular}

a) First and second refer to first and second heating curves.

\section{Water uptake measurements}

Films were prepared by solution casting of $10 \mathrm{wt} .-\%$ chloroform solutions of the polymer onto silanized dishes. The relative water uptake, defined as the difference between dry and wet weight divided by the dry weight, was determined gravimetrically after immersing films of $300 \mu \mathrm{m}$ thickness in water. Equilibrium readings were obtained within ten minutes. No further significant water uptake was found after $24 \mathrm{~h}$. The water uptake of the polymers LAS1 and DAS1 was not determined, because these polymers, having a PEG content of $82 \mathrm{wt} .-\%$, were found to be soluble in water at low concentrations. For the blend LDAS 2 only a few data points could be collected, because the samples had a tendency to fracture after swelling. Water uptake was solely 
determined by the PEG content of the polymer and equilibrium readings of 35 and $125 \mathrm{wt} .-\%$ water uptake, with a maximum error margin of 10 percent of the value, were obtained for polymers containing 28 and 55 wt.-\% PEG blocks, respectively.

\section{Conclusions}

Well defined ABA triblock copolymers of PEG (B) and PLLA (A) copolymers were prepared by polymerization of L-lactide in refluxing toluene using $\mathrm{Sn}(\mathrm{Oct})_{2}$ as a catalyst and the $-\mathrm{OH}$ end groups of PEG6000 as macroinitiators. Solution polymerization has the advantage of yielding polymers with a composition close to the composition expected from the feed contents, without discoloration at a low polymerization temperature and unimodal molecular weight distributions. Stereocomplex formation of poly(lactides) occurs readily in blends of these ABA block copolymers, but does not alter the water uptake properties of these polymers.

These investigations were supported by the Netherlands Foundation for Chemical Research with financial aid from the Netherlands' Technology Foundation.

1) M. Vert, S. M. Li, G. Spenlehauer, P. Guerin, J. Mat. Sci.: Mat. Med. 3, 432 (1992)

2) J. M. Schakenraad, P. J. Dijkstra, Clinical Materials 7, 253 (1991)

3) A. S. Sawhney, C. P. Pathak, J. A. Hubbel, Macromolecules 26, 581 (1993)

4) A. M. Reed, D. K. Gilding, Polymer 22, 499 (1981)

5) Y. Ikada, K. Jamshidi, H. Tsuji, S.-H. Hyon, Macromolecules 20, 906 (1987)

6) G. L. Loomis, J. R. Murdoch, K. H. Gardner, Polym. Prepr. (Am. Chem. Soc., Div. Polym. Chem.) 31 (2), 55 (1990)

7) H. Tsuji, F. Horii, S.-H. Hyon, Y. Ikada, Macromolecules 24, 2719 (1991)

8) H. Tsuji, Y. Ikada, Macromolecules 26, 6918 (1993) and references cited therein

9) N. Yui, P. J. Dijkstra, J. Feijen, Makromol. Chem. 191, 481 (1990)

10) S. J. McLain, N. E. Drysdale, Polym. Prepr. (Am. Chem. Soc., Div. Polym. Chem.) 33, 463 (1992)

11) D. Cohn, H. Younes, J. Biomat. Mat. Res. 22, 993 (1992)

12) L. Youxin, T. Kissel, J. Controlled Release 17, 247 (1993)

13) Z. Jedlínski, P. Kurcok, W. Walach, H. Janeczek, I. Radecka, Makromol. Chem. 194, 1681 (1993)

14) P. Cerrai, M. Tricoli, Makromol. Chem., Rapid Commun. 14, 529 (1993)

15) H. R. Kricheldorf, J. Meier-Haack, Makromol. Chem. 194, 715 (1993)

16) D. S.-G. Hu, H. J. Liu, J. Appl. Polym. Sci. 51, 473 (1994)

17) H. R. Kricheldorf, C. Boettcher, K.-U. Tönnes, Polymer 33, 2817 (1992)

18) W. M. Stevels, M. J. K. Ankoné, P. J. Dijkstra, J. Feijen, Macromol. Chem. Phys. 196, 1153 (1995) 